\title{
Design and Fabrication of a Novel Core-Suspended Optic Fiber for Distributed Gas Sensor
}

\author{
Tao ZHANG ${ }^{1,2,3^{*}}$, Lijia MA ${ }^{1,2}$, Hongbo BAI ${ }^{1,2}$, Chengguo TONG ${ }^{1,2}$, Qiang DAI ${ }^{1,2}$, \\ Chong $\mathrm{KANG}^{2,3}$, and Libo YUAN ${ }^{1,2}$
}

\begin{abstract}
${ }^{1}$ Key Laboratory of in-Fiber Integrated Optics, Ministry Education of China, Harbin Engineering University, Harbin, 150001, China

${ }^{2}$ College of Science, Harbin Engineering University, Harbin, 150001, China

${ }^{3}$ Postdoctoral Research Station of Mechanical Engineering, College of Mechanical and Electrical Engineering, Harbin Engineering University, Harbin, 150001, China
\end{abstract}

*Corresponding author: Tao ZHANGＥ-mail: taozhang@hrbeu.edu.cn

\begin{abstract}
We designed a novel core-suspended capillary fiber that the core was suspended in the air hole and close to the inner surface of the capillary, and experimentally demonstrated its fabrication technology. In addition, a method for linking a single mode fiber and a core-suspended fiber was proposed based on splicing and tapering at the fusion point between the two fibers. By combining with the optical time domain reflectometer technology, we constructed a distributed gas sensor system to monitor greenhouse gas based on this novel fiber.
\end{abstract}

Keywords: Hollow fiber, core-suspended, linking, distributed gas sensor

Citation: Tao ZHANG, Lijia MA, Hongbo BAI, Chengguo TONG, Qiang DAI, Chong KANG, et al., "Design and Fabrication of a Novel Core-Suspended Optic Fiber for Distributed Gas Sensor,” Photonic Sensors, 2014, 4(2): 97-101.

\section{Introduction}

Nowadays, Global warming is a serious challenge to humanity and is also one of the acknowledged global environmental issues for the international community $[1,2]$. The most important reason for global warming is the exhausted greenhouse gas including $\mathrm{CO}_{2}, \mathrm{~N}_{2} \mathrm{O}, \mathrm{CH}_{4}$, and $\mathrm{O}_{3}$. Among of them, the gases of $\mathrm{CO}_{2}$ and $\mathrm{CH}_{4}$ exist both the largest emission and the biggest influence. Therefore, it is very import to detect and measure the two gases. $\mathrm{CO}_{2}$ and $\mathrm{CH}_{4}$ gas sensors based on the optical principle are very suitable to monitor a variety of explosive gases such as carbon dioxide [3-5]. This kind of sensor has the advantages of high accuracy, good selectivity, high reliability, independence on the oxygen, and long life etc. So, there are some optic fiber-based sensors developed for air pollution monitoring.

In this paper, we designed a novel core-suspended capillary fiber (labeled as CSCF) that the core was suspended in the air hole and close to the inner surface of the capillary, and experimentally demonstrated its fabrication technology including a modified "core-suspendedin-tube" preform fabrication technique and fiber drawing technique with the control of the internal pressure. Then, combined with the optical time domain reflectometer (OTDR) technology, we employed this fiber to construct a new-type

Received: 20 December 2013 / Revised version: 8 January 2014

(C) The Author(s) 2014. This article is published with open access at Springerlink.com

DOI: $10.1007 / \mathrm{s} 13320-014-0171-6$

Article type: Regular 
distributed gas sensor system to monitor greenhouse gas.

\section{Experiments}

\subsection{Structure and design of fiber}

The configuration of the CSCF is illustrated in Fig. 1. It is composed of three parts including a central circular hole with the diameter of about $62.5 \mu \mathrm{m}$, an $8-\mu \mathrm{m}$ suspended core, and a $125-\mu \mathrm{m}$ diameter silica cladding. The refractive indexes of the cladding, the core, and hole are expressed by $n_{s}$, $n_{c}$, and $n_{h}$, respectively, and $n_{c}>n_{s}>n_{h}=1$. Because the core is suspended on the inner surface of the fiber central hole, it will increase the contact area between the internal gas in hole and the fiber; moreover, the evanescent wave can be transmitted into the central hole through the core. When there are varieties of gases in the central hole, the evanescent wave will be effectively absorbed by the substance. Thus, this will cause light transmission loss in the core due to the presence of the gases. So, this kind of fiber may be predicted to make the biosensor based on the evanescent field.

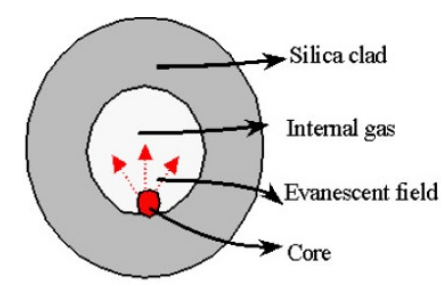

Fig. 1 Configuration of the CSCF.

\subsection{Fiber Fabrication}

The fabrication technology of the CSCF includes two steps: preform preparation and fiber drawing to the traditional commercial optical fiber. The optical fiber preform is the original product fabricated according to the fiber design and basically determines the characteristics and quality of the finished optical fiber. During the process, the core-suspended preform is fabricated by using a modified "core-suspended-in-tube method" [6]. The complex preform comprises a core rod and a silica tube, as shown in Fig. 2. The inner and outside diameters of the silica tube are $9.4 \mathrm{~mm}$ and $13 \mathrm{~mm}$, respectively. The core rod diameter is $1 \mathrm{~mm}$ with the cladding/core ratio of 2:1. The core rod is manufactured by the modified chemical vapor deposition technique and a custom-made fiber drawing tower. Firstly, the core rod is horizontally inserted into a silica tube. Under the gravity, the core rod can automatically be fixed on the inner surface of the silica tube along the axis. Then, by heating with the oxyhy-drogen burner and horizontally moving, the core rod can be welded tightly on the wall of the silica tube, and a complex preform is formed. Finally, the one end of the complex will be tapered into a neck shape to avoid dust and impurity contamination.

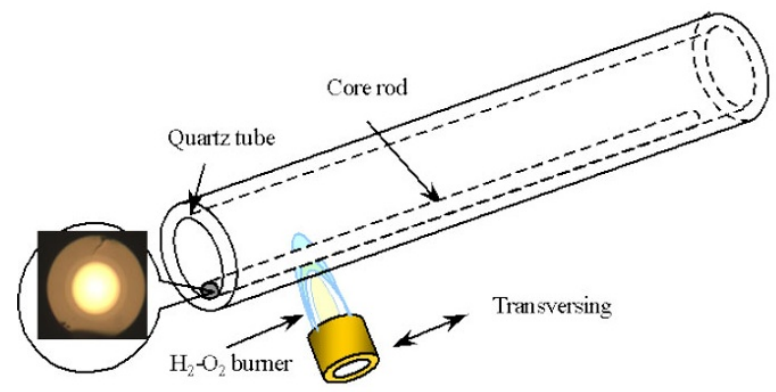

Fig. 2 Sketch map of fabrication process with the core-suspended preform.

The CSCF is fabricated from the complex by utilizing a custom-made fiber drawing tower. In this process, the preform is secured with the chuck at the top of the tower, and one end is attached to the gas injected system. When it is heated to approximately $2000{ }^{\circ} \mathrm{C}$, the preform is sufficiently softened, and the initial lump of the preform is dropped. Thus, the CSCF is drawn with the aid of the tractor mechanism.

Furthermore, the gas pressure in the silica tube and drawing temperature are both very important factors which determine the final shape of the fiber core. As shown in Fig. 3, a pressure controller is employed in the drawing tower. The pressure controller can provide the positive pressure in the silica tube by using the pneumatic device, which can 
not only avoid collapse of the silica tube at higher drawing temperature but also keep the shape of the core rod. The pressure value changes from 0 to $+1000 \mathrm{~Pa}$ depending upon the drawing process and the required core dimensions. When the outer diameter of the fiber reaches to about $125 \mu \mathrm{m}$ during the drawing process, the fiber will pass through a die for coating and curing, as shown in Fig. 3. The CSCF is finally coated with ultrviolet (UV) curable acrylate in order to maintain the pristine surface.

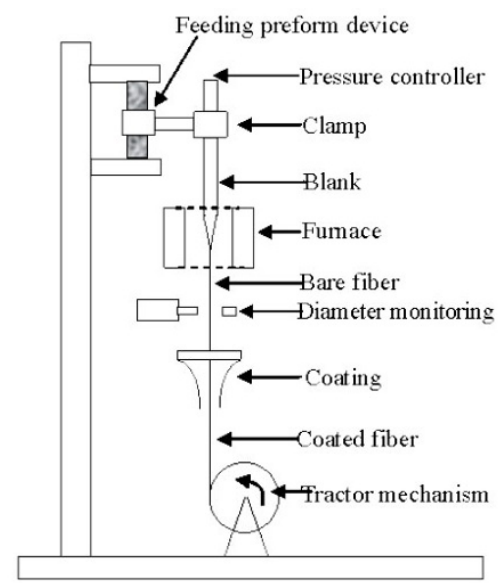

Fig. 3 Drawing tower setup with the pressure controller.

\subsection{Refractive measurement}

The refractive index profile of the fibers is performed using a refracted near-field technique. Prior to examination, the fiber coating is peeled, and the end glossily is cut with the electric fiber cleaver. Then, the fiber is put into a sample cell filling with index matching liquid. The uncovered fiber is investigated with an S14 refractive index profiler (manufactured by PK technology).

\subsection{Distributed gas sensor integration}

In order to achieve this fiber-based gas sense, it is very important to joint the CSCF with the single mode fiber together, firstly. The tapering process [7, 8] of the single mode fiber and CSCF experimental setup are shown in Fig. 4. A laser diode with a wavelength $1310 \mathrm{~nm}$ is used as the light source and connected with the single mode fiber, and an infrared charge coupled device (CCD) camera is amounted on the output end of the CSCF to monitor the output optical power. The linking and tapering process is described as follows: firstly, a standard single mode fiber and the CSCF are fused by using a fiber fusion splicer (type KL-260B), and there is no light can be seen from the output end of the CSCF. After the two fibers have been fused together, make a mark at the splicing point and then move the linked fiber to the fiber tapering system for heating and stretching. In the period of heating, in order to make a smooth bi-tapered fiber coupler at the two fiber joint parts, the center of the $\mathrm{H}_{2}-\mathrm{O}_{2}$ burner should move to the standard single mode fiber a small distance, thus the center of the high-heating zone will surround the single mode fiber, and more quantity of heat could be absorbed by the single mode fiber than the CSCF.

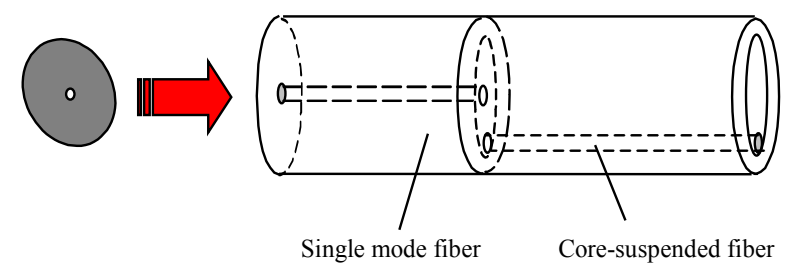

(a) Schematic diagram of fusing between the single mode fiber and CSCF

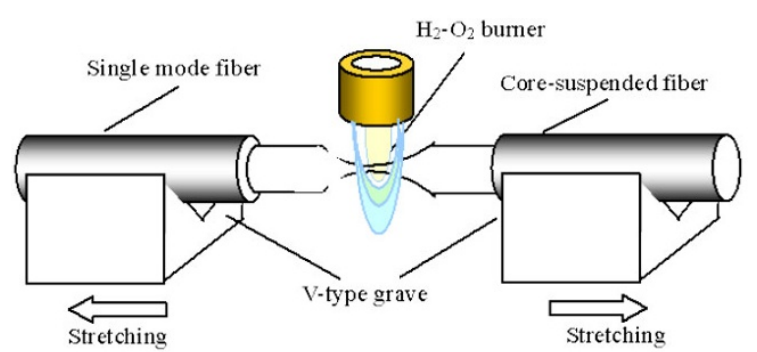

(b) Tapering experimental setup of the single mode fiber and CSCF

Fig. 4 Tapering process of the single mode fiber and CSCF.

During the heating and stretching of the two fiber splicing points, the output optical power distributed in the annular core of the capillary fiber end is monitored by the CCD camera. The tapering process will stop until the output power gradually increases to the highest value. The possible reason is that the two modes (radiation-to-guiding mode) between the single mode fiber and the inner annular core capillary fiber are gradually matched each 
other.

Next, some distributed micro-holes are bored on the surface of the CSCF at an interval of several meters using the deep-ultraviolet $(157 \mathrm{~nm})$ micromachining method in order to introduce and expel detected gas into the CSCF. Gas sensing based on the CSCF can be fulfilled in the following process (as shown in Fig. 5). At first, the end of the tapered simple mode fiber is linked to the OTDR, a laser with the wavelength $1310 \mathrm{~nm}$ is injected into the simple mode fiber and transmitted into the CSCF through the couple zone, and then there are always evanescent wave present. When the CSCF with holes meets to the $\mathrm{CO}_{2}$ gas, the evanescent wave will interact with the penetrated gas through the clad and lead to absorption, which increases the fiber loss at this point. Thus, the returned signal by Rayleigh backward scattering will be collected by a detector in the OTDR. Since the absorption is related to the gas content at different places, the returned signal can diagnose the gas leak point position in underground pipelines and other environments.

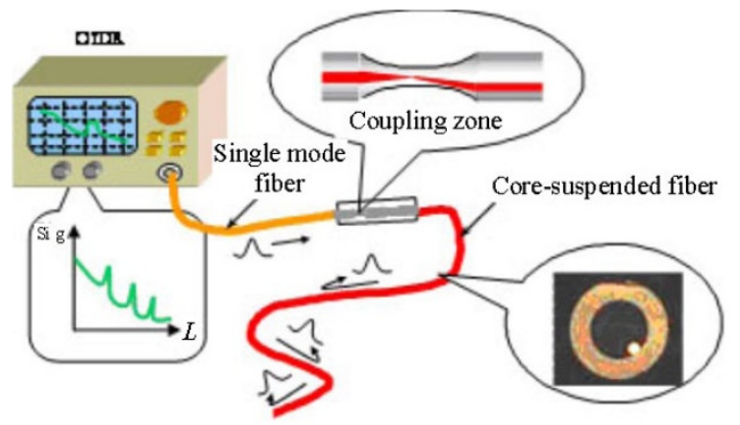

Fig. 5 Distributed fiber-optic gas sensor based on the core-suspended optic fiber.

\section{Results and discussion}

Figure 6 shows the cross section photos of the $\mathrm{CSCF}$ at different temperature and pressure control. One can see when the fiber is drawn at lower temperature and without gas pressure control, it is easy to keep the shape of the suspended core due to the small surface tension effect at the interface of the core rod and tube. When the drawn temperature rises, the core will become elliptic and even be embedded into the tube wall owing to surface tension action, as show in Figs. 6(a) and 6(b). It is the same as that of injected gas pressure [shown in Figs. 6(c) and 6(d)]. So, the proper pressure in the silica tube and lower drawing temperature are both important factors to control the shape of the fiber core.

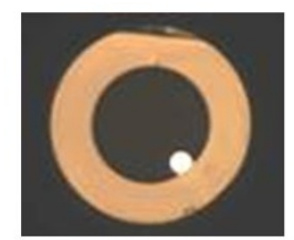

(a)

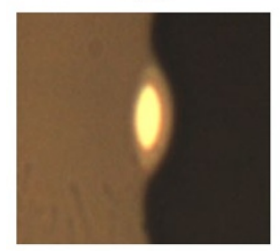

(c)

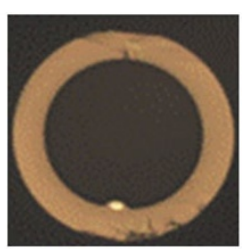

(b)

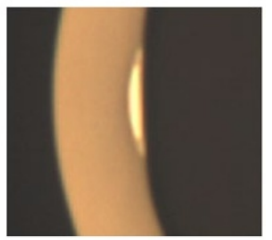

(d)
Fig. 6 Cross section pictures of fibers drawn at (a) $1900{ }^{\circ} \mathrm{C}$ without internal presure, (b) $2000^{\circ} \mathrm{C}$ without pressure, (c) $2000^{\circ} \mathrm{C}$ with pressure $100 \mathrm{~Pa}$, and (d) $2000^{\circ} \mathrm{C}$ with pressure $500 \mathrm{~Pa}$.

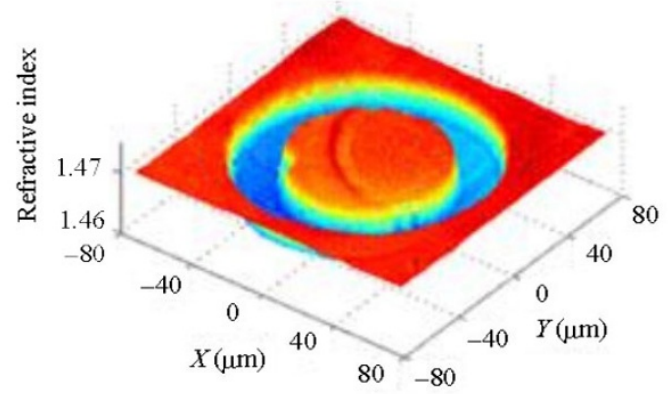

Fig. 7 3-D scan mapping of the refractive index profile of the CSCF.

The refractive index profile of the CSCF is plotted in Fig. 7. It can be seen that the air hole diameter is about $62.5 \mu \mathrm{m}$, and the suspended core is laid on the periphery of the clad with a diameter about $7 \mu \mathrm{m}$.

\section{Conclusions}

In conclusion, we have designed and manufactured a novel core-suspended optic fiber for the distributed gas sensor. By means of a modified "core-suspended-in-tube" method, the coresuspended preform was fabricated, and the corresponding fiber was also drawn on the condition 
of proper temperature and pressure controlling.

Moreover, we have also proposed a simple linking coupling method between the simple mode fiber and core-suspended optic fiber, and constructed a new-type distributed gas sensor system to monitor greenhouse gas based on this novel fiber.

\section{Acknowledgment}

This work was supported by the Key Laboratory Program for in-Fiber Integrated Optics of the Education Ministry of China, partially supported by the National Nature Science Foundation of China (No. 41174161) and by China Postdoctoral Science Foundation Grant (2013M531013).

Open Access This article is distributed under the terms of the Creative Commons Attribution License which permits any use, distribution, and reproduction in any medium, provided the original author(s) and source are credited.

\section{References}

[1] Intergovernmental Panel on Climate Change (IPCC),
Climate change 2007: summary for policymakers. Cambridge, UK: Cambridge University Press, 2007: 125.

[2] S. Holloway, J. M. Pearce, V. L. Hards, et al. "Natural emissions of $\mathrm{CO}_{2}$ from the geosphere and their beating on the geological storage of carbon dioxide," Energy, 2007, 32(7): 1194-1201.

[3] Y. L. Hoo, W. Jin, C. Shi, H. L. Ho, D. N. Wang, and S. C. Ruan, "Design and modeling of a photonic crystal fiber gas sensor," Applied Optics, 2003, 42(18): 3509-3515.

[4] C. Singh, Y. Shibata, and M. Ogita, "A theoretical study of tapered, porous clad optical fibers for detection of gases," Sensors and Actuators B: Chemical, 2003, 92(1-2): 44-48.

[5] X. Y. Bao, "Optical fiber sensors based on Brillouin scattering," Optics and Photonics News, 2009, 20(9): 40-46.

[6] T. Miyashita, T. Edahiro, S. Takahashi, M. Horiguchi, and K. Masuno, "Eccentric-core glass optical waveguide," Journal of Applied Physics, 1974, 45(2): 808-809.

[7] L. B. Yuan, X. L. Zhu, T. Zhang, F. J. Tian, Q. Dai, and J. Yang, "Capillary optical fiber linking approach for biosensors," in Proc. SPIE, vol. 7503: pp. 750372 , 2009.

[8] L. B. Yuan, Z. H. Liu, J. Yang, and C. Y. Guan, "Bi-tapered fiber coupling characteristics between single mode single-core fiber and multi-core fiber," Applied Optics, 2008, 47(18): 3307-3312. 